Einige kritische Untersuchungen über die quantitativen Fällungsverhältnisse versehiedener Proteïnfällungsmittel.

Von

\title{
H. Schjerning.
}

(Mitheilung aus dem chemischen Laboratorinm Neu-Carlsberg.)

Wie es Jedermann, der sich irgendwie mit den Proteïnstoffen and. deren analytischem Verhalten beschäftigt, bekannt sein wird, ist die Litteratur reich an Methoden zur Ausfällung dieser Stoffe. Oft wurde das vorgeschlagene Proteïnfällungsmittel nur in seinem Verhalten gegenüber einer ganz bestimmten, oder doch nur gegenüber verhältnissmässig wenigen verschiedenartigen Proteïnlösungen untersucht and geprüft, und manchmal weichen die Angaben verschiedener Forscher in irgend einem Punkte wesentlich von einander $a b$.

Meiner Auffassung und Erfahrung nach muss man die Aufmerksamkeit ganz besonders auf 3 Punkte richten, wenn es sich um die Beurtheilung einer quantitativen Proteïnfällong handelt, nämlich:

I. dass die Fällung von einer solchen Beschaffenheit sein muss, dass schon das blosse Auge über ihre etwaige Brauchbarkeit Auskunft geben kann, oder, wie ich dies früher ausgedrückt habe, dass Filtrat und Waschflüssigkeit ganz klar sein müssen;

II. dass in dem Niederschlag entweder alle Proteïngruppen oder nur ganz bestimmte enthalten sein müssen, und zwar soll dies nicht nur an einer bestimmten, einzigen Proteinnlösung geprüft sein, sondern es ist so zu verstehen, dass diese Reaction sich gegenüber einer Reihe verschiedener und verschiedenartiger Proteinlösungen wiederholt.

III. Die Fällung darf keine anderen stickstoffhaltigen organischen Verbindungen enthalten als gerade die Proteine - also keine Amin-Amidverbindungen, Fleischbasen, Xanthinverbindungen u. s. w.

Fresenins, Zeitschrift f. analyt. Chemie. XxxIx. Jahrgang. 9. Heft. 
546 Schjerning: Einige kritische Untersuchungen über die quantitativen

Auf den ersten Punkt werde ich nicht näher eingehen. da es sich wohl von selbst ergibt, dass das Filtrat von einer quantitativen Fällung immer klar sein muss, wenn man mit Aussicht auf ein zuverlässiges Resultat die Bestimmung weiter zu führen berechtigt sein will.

Das unter $»$ Punkt II $\ll$ Angeführte habe ich in meinen früheren Publicationen ${ }^{1}$ ) zu beweisen gesucht, bevor ich meine analytische Methode zur quantitativen Trennung der verschiedenen Proteïngruppen ${ }^{2}$ ), wenn diese in einer gemischten Lösung enthalten sind, veröffentlichte. Ich bin jedoch immer dariuber im Klaren gewesen, dass mehrere Einwendungen gegen mein Verfahren erhoben werden können; aber einen ganz unumstösslichen Beweis für oder wider zu erbringen, dürfte, glaube ich, im Augenblicke kaum möglich sein.

Wenn Laszczynski ${ }^{3}$ ) sich über meine Methode folgendermaassen ausspricht: Schjerning hat eine eigenartige und neue Methode ausgearbeitet, indem er von der Beobachtung ausging, dass Zinnchlorür, Bleiacetat, Ferriacetat und Uranacetat bei parallelen Fällungen in der Würze steigende Stickstoffmengen in den Niederschlägen geben. Er nahmalso an, dass Zinnchlorür:Albumin, Bleiacetat: Albumin + Denucleïn, Ferriacetat: Albumin + Denuclein + Propepton, Uranacetat : Albumin + Denuclein + Propepton + Pepton ausfällt, wobei vorausgesetzt wurde, dass das nächstfolgende Reagens auch mit den vom vorhergehenden gefällten Substanzen Ferbindungen eingehe, eine Behauptung, welche nach unseren Beobachtungen kaum zutreffen düfte «, so muss ich ihm erwidern, dass er übersehen haben muss, dass in meiner ersten Abhandlung in der möglichst directen Weise nachgewiesen wurde, dass ich $\mathrm{n}$ icht von der progressiven Fällang der verschiedenen Proteïngruppen als etwas Gegebenem ausgegangen bin, sondern gerade im Gegentheil durch meine Versuche zeigte, dass diese progressive Fällung stattfindet. In meinen späteren Publicationen, welche indess Laszczynski nicht zu Gesicht bekommen hat und deren er deshalb nicht erwähnt, komme ich erst zu dem eigentlichen Material,

1) Diese Zeitschrift $\mathbf{3 3}, 263 ; \mathbf{3 4}, \mathbf{1 3 5} ; \mathbf{3 5}, 285 ; \mathbf{3 6}, 643 ; \mathbf{3 7}, 73$.

2) Ibid. 37, 413.

a) Zeitschr. f. d. gesammt. Brauw. 22, 123. 
welches über die Natur und Classification der einzelnen Proteïnfällungen Licht verbreiten kann. Was mich bei diesen Untersuchungen besonders angeleitet hat, ist die nevere Auffassung der wirklichen Peptone als Proteïnstoffe, welche sich weder aus neutralen noch aus sauren Lösungen durch Sättigung mit neutralen, leicht löslichen Salzen aussalzen lassen - ich habe Magnesiumsulfat benutzt. Wird dagegen eine vorher mit circa $0,5 \%$ freier Essigsäure vermischte Proteünlösung mit einem leicht Iöslichen Salze $\left(\mathrm{Mg} \mathrm{SO}_{4}\right)$ gesättigt, so werden ausschliesslich diejenigen Proteïnstoffe ausgefällt, welche unter die Gruppe der Albumine und Propeptone (Albumosen) gezählt werden können. Da das Verhältniss der Magnesiumsulfatfällung zu den übrigen Fällungen ausserdem durch experimentelle Versuche vollständig beleachtet ist, ${ }^{1}$ ) glaube ich auch fernerhin - jedenfalls bis ein auf Versuche gestützter Gegenbeweis vorliegt - die Richtigkeit der von mir vorgeschlagenen Proteïntrennungsmethode behaupten za dürfen. Im Uebrigen werde ich diese Sache später wieder berühren.

Die vorliegrende kleine Arbeit bezweckt jedoch zunächst, was ich in dem obigen dritten Punlte (III) ausgedrückt habe, nämlich zu untersuchen, wie die von mir vorgeschlagenen 6 Proteïnfäliungsmittel, wie auch einige andere neuere und altere, sich in analytischer Beziehung gegenüber organischen Stickstoffverbindungen von nicht-proteïnartiger Natur -- Amin-Amidsäuren, Fleischbasen u. s. w. - verbalten. Ueber diese Untersuchungen werde ich im folgenden Abschnitte Bericht erstatten.

\section{I.}

Ehe ich dazu übergehe, die bei diesen Versuchen gewonnenen Resultate mitzutheilen, muss ich nothwendigerweise mit einigen Worten die Ausführung der cinzelnen Fällungen erörtern.

Mit Rücksicht auf die Fällungen mit Zinnchlorür, Quecksilberchlorid, Bleiacetat, Ferriacetat, Uranacetat und Magnesiumsulfat wird ein Hinweis anf meine früher gegebene Beschreibung der praktischen A usführung dieser Fällungen ${ }^{2}$ ) genügen, indem es nur noch nothwendig ist hinzuzufügen, dass alle diese Versuche natürlich so vorgenommen wurden, als ob mit aschenfreien Proteïnlösungen gearbeitet würde. Da

1) Diese Zeitschrift 35, 291-93.

2) Diese Zeitschrift $\mathbf{3 7}, 413$. 
ich indess früher ${ }^{1}$ ) nachgewiesen habe, dass Uranacetat eine gewisse Menge Ammoniak ausfällen kann, wenn die Flässigkeit gleichzeitig freie Phosphorsäure oder lösliche Phosphate enthält, war natürlich die Möglichkeit nicht ausgeschlossen, dass Uranacetat unter ähnlichen Verhältnissen auch andere, ausserhalb der Gruppe der Proteinstoffe stehende stickstoffhaltige Verbindungen rein organischer Natur auszufällen vermöchte.

Mit Rücksicht auf diese Möglichkeit wurde ausser der normalen Uranfällung noch eine andere Uranfällung vorgenommen, bei welcher die zum Versuche verwendete Substanzmenge vorher mit einer circa 0,04g Phosphorsäure entsprechenden Menge gewöhnlichen Natriumphosphats versetzt war. Die auf diese Weise hervorgebrachte Uranfällung wurde 4 bis 5 mal mit einer kalten 1-2 procentigen Uranacetatlösung ausgewaschen, darauf wurden Filter und Niederschlag in einen 1/4 Liter-Kolben übertragen, mit $50 \mathrm{cc}$ Magnesiamilch - $11 \mathrm{~g}$ gebrannte Magnesia in zwei Litern Wasser - versetzt und auf einer Asbestplatte über einer schwachen Gasflamme beinahe, aber doch nicht vollständig, zur Trockne abgedampft, und dann der Rest nach Kjeldahl's Methode behandelt.

$50 \mathrm{cc}$ der oben genannten Magnesiamilch werden an Sättigungsfähigkeit circa $125 \mathrm{cc} 1 / 10$ Normalsäure entsprechen - einer Säuremenge, welche auch-nicht anuähernd in einer nach meiner Methode ausgeführten Uranfällung enthalten sein kann. Uebrigens kann man sich leicht davon überzeugen, dass der Eindampfungsrest mit einem Tropfen Phenolphtalein alkalische Reaction zeigt. Das Eindampfen mittelst Kalkmilch vorzunehmen, ist nicht rathsam, da diese Base unzweifelhaft so stark ist, dass sie gewisse Proteïnstoffe theilweise za zersetzen vermag, was bei Anwendung von Magnesiamilch nicht geschieht. Dass die Magnesiamilch andererseits die gesammte ausgetällte Ammoniakmenge auszutreiben im Stande ist, geht aus den nachfolgenden Versuchen hervor.

Ausser den oben angeführten 7 Fällungen wurden auch mit Brom, mit Gerbsänre, mit Stutzer's Reagens und mit Phosphorwolframsäure Fällungen vorgenommen.

Die Bromfällung wurde auf die von Allen und Searle ${ }^{2}$ ) beschriebene Weise ausgeführt indem die Flüssiglkeit mit Salzsäure an-

1) Diese Zeitschrift 23 , 287; siehe auch Arendt und Knop, Chem. Centralbl. 1856 , s. 769 .

2) The Analyst 22, 258; oder Chem. Centralbl. 1897, II. S. 1163; siehe auch Wiley. Chem. News 80, 88; oder Chem. Centralbl. 1899, II, S. 586. 
gesäuert, mit Ueberschuss von Bromwasser versetzt und unter Umrühren $1 / 2-1$ Stunde bei gewöhnlicher Zimmertemperatur stehen gelassen wurde. Der Niederschlag wurde dann auf einem Filter gesammelt und 2-3 mal mit verdünntem Bromwasser ausgewaschen. Da es sich indessen herausstellte, dass der Niederschlag sich oft gar nicht sammeln liess oder sogar gänzlich in der Waschflüssigkeit gelöst wurde, wurden sowohl die Fällungsflüssigkeit als auch die Waschflüssigkeit stetig mit einer geringen Menge Magnesiumsulfat versetzt.

Die Gerbsäurefällung wird bei gewöhnlicher Temperatur vorgenommen indem die Fällungsflüssigkeit zuerst mit ein wenig Magnesiumsulfat versetzt, mit Essigsäure angesäuert und dann mit einer genügenden Menge 10 procentiger Gerbsäurelösung versetzt wird - ein grösserer Ueberschuss ist nach Möglichkeit zu vermeiden. Die Fällung wird etwa 2 Stunden lang stehen gelassen und der angesammelte Niederschlag 2 bis 3 mal mit kaltem Wasser ausgewaschen.

Die Fällung mit Stutzer's Reagens. Das Reagens wurde nach Fassbender's Methode ${ }^{1}$ ) dargestellt. Durch vorläufige Versuche mit Asparagin, Leucin and Tyrosin wurden die Fällungsbedingungen festgesetzt. Diese vorläufigen Versuche ergaben folgende Resultate, berechnet in Procenten der gesammten Aminsäuremenge.

Tabelle I.

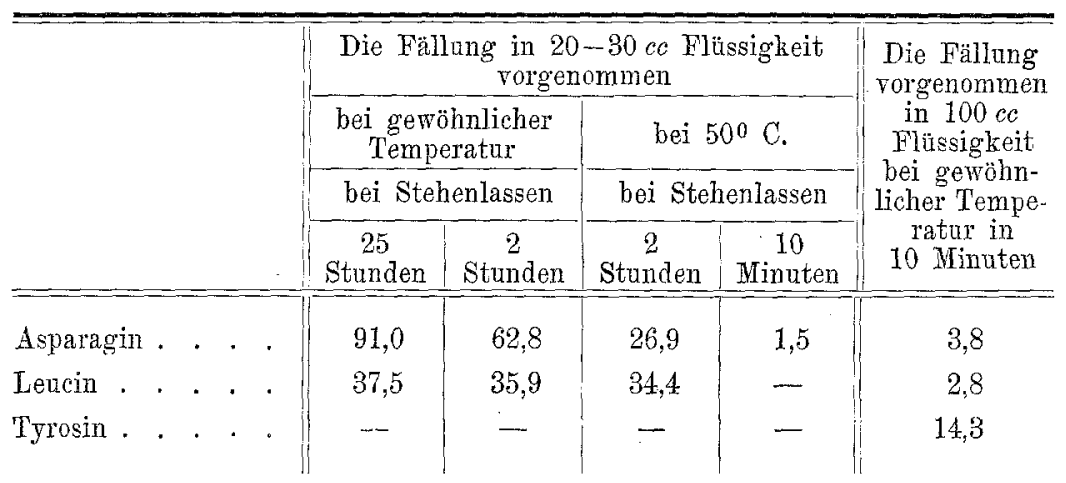

1) Berichte der deutschen chem. Gesellschaft zu Berlin 13, 1822 und diese Zeitschrift 20, 588 . 
550 Schjerning: Einige kritische Untersuchungen über die quantitativen

Bereits aus diesen wenigen Versuchen konnten die Fällungsbedingungen, wernn man eine Ausfällung von Aminsäuren vermeiden will, leicht aufgestellt werden. Ich nahm daher Stutzer's Fällung stets in $100 \mathrm{cc}$ Flüssigkeit vor, indem ich 10 MLinuten lang im Wasserbad bei $50^{\circ} \mathrm{C}$. stehen liess und recht häufig umrührte. Nach dem Verlauf der 10 Minuten wurde der Niederschlag auf einem Filter gesammelt und $4-5$ mal mit kaltem Wasser ausgewaschen.

Die Phosphorwolframsäurefällung wurde in einer Flüssigkeit vorgenommen, welche circa $2 \%$ freie Schwefelsäure nebst einem Ueberschusse von Phosphorwolframsäure enthielt. Es wurde eine Fällung bei gewöhnlicher Temperatur und eine unter Kochen vorgenommen, indem der Niederschlag bei lotzterer, gleich nachdem die Flüssigkeit $z u$ kochen angefangen hatte, auf einem Filter gesammelt wurde. Beide Fällungen wurden $2-3$ mal mit kalter etwa 2 procentiger Schwefelsäure ausgewaschen.

Was die bei den Versuchen benutzten stickstoffhaltigen organischen Stoffe angeht, ist nur zu bemerken, dass stets mit E. Merck's reinen Präparaten gearbeitet wurde. Nur das Leucin und die Glutaminsäure musste ich weiter reinigen. Das benutzte Arginin wurde gemäss der von $\mathrm{Hedin}$ gegebenen Vorschrift $^{1}$ ) hergestellt, indem ich jedoch zur Trennung der gebildeten Aminsäuren Kossel's Nethode ${ }^{2}$ ) anwendete. Mein Argininpräparat war rein weiss, krystallinisch und enthielt 31,9\% Stickstoff, während die Formel des Arginins $\left(\mathrm{C}_{6} \mathrm{H}_{14} \mathrm{~N}_{4} \mathrm{O}_{2}\right) 32,18 \%$ verlangt.

In der nachfolgenden Tabelle sind meine Versuchsresultate zusammengestellt, indem die Zahlen stets angeben, wie viele Procente der gesammten Stickstoffmenge von dem betreffenden Fällungsmittel ausgefällt werden. Die Titrirung wurde jodometrisch vorgenommen, und der zulässige Fehler auf dieselbe Weise wie in meinen früheren Arbeiten berechnet.

1) Zeitschrift f. physiol. Chemie $\mathbf{2 1}, 160$.

2) Ibid. 25, 165 . 
Tabelle II.

\begin{tabular}{|c|c|c|c|c|c|c|c|c|c|c|c|c|c|c|}
\hline & \multirow{2}{*}{ 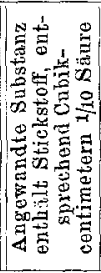 } & \multirow{2}{*}{ 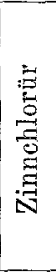 } & \multirow{2}{*}{ 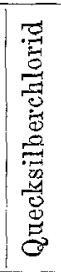 } & \multirow{2}{*}{ 离 } & \multirow{2}{*}{ 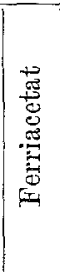 } & \multicolumn{2}{|c|}{$\begin{array}{l}\text { Uran- } \\
\text { acetat }\end{array}$} & \multirow{2}{*}{ 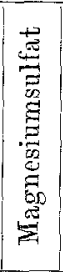 } & \multirow{2}{*}{ 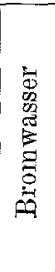 } & \multirow{2}{*}{ 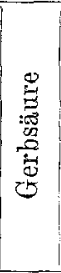 } & \multirow{2}{*}{ 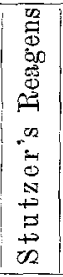 } & \multicolumn{2}{|c|}{$\begin{array}{l}\text { Phos- } \\
\text { phor- } \\
\text { wolfram- } \\
\text { säure }\end{array}$} & \multirow{2}{*}{ 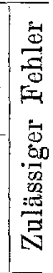 } \\
\hline & & & & & & ohne & $\begin{array}{c}\text { mit } \\
\mathrm{P}_{2} \mathrm{O}_{5}\end{array}$ & & & & & 蓔 & 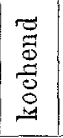 & \\
\hline mmor & 24,4 & - & 12,3 & - & - & - & - & - & - & - & - & $x$ & $\times$ & 0,4 \\
\hline Jetrin & 3,9 & -1 & . & 1. & . & - & - & - & - & 15,4 & . & 69,2 & 71,8 & 2,6 \\
\hline etaïn . $\mathrm{HCl}$ & 6,8 & - & - & - & - & - & - & - & -1 & -1 & $1-$ & 38,2 & 27,9 & 1,5 \\
\hline nonin & 4,0 & - & - & $1-$ & $\cdot$ & - & - & - & 一 & - & 47,5 & 45,0 & - & 2,5 \\
\hline$\Delta 110$ & 9,1 & 26,4 & - & $3,3 \mid$ & 24,2 & 一 & - & - & - & -1 & 31,9 & $\cdot$ & - & 1,1 \\
\hline Allantoin . & 13,8 & - & - & - & . & 一 & - & - & - & -1 & 2,9 & - & - & 0,7 \\
\hline re & & - & - & 2,5 & - & 一 & 一 & - & -- & 一 & 2,5 & 5,0 & - & 1,8 \\
\hline $\operatorname{gin}$ & 15,3 & - & - & - & - & 一 & 4,5 & - & - & - & - & 2,5 & - & 0,7 \\
\hline 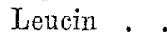 & & - & 一 & - & - & $\ldots$ & $\ldots$ & 一 & - & - & - & -1 & -1 & 1,6 \\
\hline Arginin . & 12,5 & - & 一 & - & - & - & 18,4 & - & - & - & 4,0 & 79,2 & - & 0,8 \\
\hline larnsäure . & 1,6 & - & - & - & - & - & - & -1 & - & - & 31,3 & -1 & - & 6,3 \\
\hline äure & 2 & - & - & - & . & 一 & - & 25,0 & - & - & - & - & - & 3,1 \\
\hline a. . & 2,6 & -1 & - & - & - & - & - & - & $x$ & - & - & - & - & 3,8 \\
\hline razin & 3,8 & - & 94,7 & . & - & 44,7 & 71,0 & - & 68,4 & - & - & 89,5 & 36,8 & 2,6 \\
\hline offeîn. & 15,9 & - & 4,4 & - & 1,3 & • & - & 94,3 & $x$ & $x$ & - & 91,2 & 93,7 & 0,6 \\
\hline Theobromin & 8,8 & - & -- & - & - & - & - & 5,7 & - & - & - & $x$ & - & 1,1 \\
\hline hir & 5,7 & - & $x$ & $3, \check{b}$ & 3,5 & - & - & 52,6 & 61,4 & $x$ & 3,5 & 96,5 & $98,2 \mid$ & 1,8 \\
\hline Lorphin . & 3,8 & - & - & - & - & 一 & $\cdots$ & - & 5,3 & - & - & 100,0 & 84,2 & 2,7 \\
\hline & 9,6 & - & - & - & - & - & 2,1 & 25,0 & 11,4 & $x$ & - & 101,0 & $?$ & 1,0 \\
\hline dalin . & $8, \mathbf{1}$ & - & - & - & - & - & - & 19,8 & - & 一 & - & -1 & -1 & 1,2 \\
\hline Solanin . & 1,5 & -1 & - & - & - & - & - & 66,7 & $x$ & $x$ & - & - & - & 6,7 \\
\hline
\end{tabular}

$X$ bedeutet, dass wohl eine Ausscheidung entstand, dass dieselbe aber so fein vertheilt war, dass sie sich nicht auf einem Filter sammeln liess. Wo es sich darum handelt, Proteinstoffe auszufäller, wird eine solche Ausscheidung doch zugleich mit der Proteïnfällung offenbar ein gesammtes Ganze bilden, welches sich leicht auf einem Filter sammeln lässt.

- bedeutet, dass eine dem zulässigen Fehler entsprechende Stofimenge ausgefält ist, siehe die letzte Colonne. Da dieser Werth ja einen Titrationsfehler vertreten kann, ist er nicht $\mathrm{zu}$ berücksichtigen.

? reichliche Ausscheidung, aber die Bestimmung ging verloren.

Anmerkung: In allen leeren Rubriken war das Versuchsresultat $=0$. 
552 Schjerning: Einige kritische Untersuchungen über die quantitativen

Ausser mit den in der Tabelle angeführten Stoffen wurden auch mit Kaliumnitrat ${ }^{1}$ ), Harnstoff, Glutaminsäure und Senföl Versuche angestellt; aber hei keinem dieser Stoffe wurde mit den benutzten Fällungsmitteln Fällung bewirkt.

Indem ich nun aus den in der Tabelle angeführten Zahlengrössen die Resultate ableite, werde ich mich möglichst kurz fassen. Man wird gleich auf den ersten Blick sehen, dass von keinem der hier geprüften Proteïnfällungsmittel mit unbedingter Berechtigung behauptet werden darf, dass es mit absoluter Sicherheit keine anderen stickstoffhaltigen organischen Stoffe fälle als gerade die Proteïne - ein Resultat, das an und für sich wohl kaum Jemanden in Erstaunen setzen dürfte.

Sehen wir zuerst auf meine 6 Fällungsmittel - Zinnchlorür, Quecksilberchlorid. Bleiacetat, Ferriacetat, Uranacetat und Magnesiumsulfat -, so zeigen mehrere von ihnen ein äusserst geringes Vermögen, organische Stickstoffverbindungen nicht-proteïnartiger Natur auszufällen. Von den untersuchten Stoffen fällt wämlich Zinnchlorür nur Alloxan; Quecksilberchlorid Ammoniumacetat, Piperazin und ein wenig Coffein; Bleiacetat eine geringe Menge Alloxan nebst einer äusserst geringen Menge Kreatin und Chinin; Ferriacetat Alloxan und äusserst kleine Mengen Coffë̈n und Chinin; Uranacetat bloss Piperazin; und endlich Magnesiumsulfat Hippursäure, Coffë̈n, ein wenig Theobromin, Chinin, Brucin, Amygdalin und Solanin. Da num Alloxan und Piperazin zwei Stoffe sind, welche wahrscheinlich nur äusserst selten zusammen mit den Proteïnstoffen vorkommen, erscheint. es als einigermaassen unwahrscheinlich, dass die vier Reagentien Zinnchlorür, Bleiacetat, Ferriacetat und Uranacetat der Regel nach andere Stoffe als die Proteïne ausfällen sollten; oder, mit anderen Worten, diese vier Reagentien sind als die zuverlässigsten Proteïnfällungsmittel zu betrachten, wenn es sich darum handelt, gegen Ausfällung stickstoffbaltiger organischer Stoffe nicht-proteïnartiger Natur gesichert zu sein. Etwas anders verhält es sich in Bezug auf die Reagentien Quecksilberchlorid und Magnesiumsulfat.

Wie aus der Tabelle II ersichtlich ist, fällt $\mathrm{Quecksilberch} \mathrm{lorid}$ eine recht bedeutende Menge Ammoniak aus Ammoniumacetat, was

1) Die Salpetersäurebestimmungen wurden stets nach Ulsch's Methode ausgeführt. 
natürich eine Fehlerquelle bildet, vor welcher man sich hüten muss, und zwar um so mehr, als man nicht erwarten darf, die ausgefällten Ammoniakmengen durch Eindampfung mit Magnesiamilch austreiben zu können, wie dies mit der von Uranacetat bei Anwesenheit löslicher Phosphate ausgefällten Ammoniakmenge der Fall ist. Vieles spricht indessen dafür, dass diese Ausfällung von Ammoniak durch Qnecksilberchlorid bei Weitem nicht so leicht und reichlich von statten gehen wird, wie der angestellte Versuch zeigt; die Fällung tritt pämlich nur dann ein, wenn das Ammoniak an organische Säuren gebunden ist, und nicht zugleich äquivalente Mengen von Chloriden zugegen sind. Besonders wirksam ist Ammoniumchlorid.

Löst man zum Beispiel äquivalente Mengen von Ammoniumacetat und Ammoniumchlorid in Wasser auf und fügt einen Ueberschuss von Quecksilberchlorid hinzu, dann zeigt sich beim Stehenlassen gar keine Ausscheidung. Auch die Chloride der leichten Metalle wirken auf dieselbe Weise, doch weniger energisch als das Ammoniaksalz.

Wird nach meiner Methode mit einer Proteïnlösung gearbeitet, von welcher $10 c c$ etwa eine $5 c c 1 / 10$ Normalsäure entsprechende gesammte Stickstoffmenge enthalten, und werden für die Quecksilberfällung $25 c c$ abpipettirt, so wird, unter der Voraussetzung, dass die gesammte Stickstoffmenge als Ammoniak vorhanden ist, eine Zngabe von $0,067 g$ Ammoniumchlorid der Ausfällung von Ammoniak ganz vorbeugen. Dieses ist ein sehr drastisches Beispiel.

Bei meinen praktischen Versuchen habe ich noch nie einem Falle gegenüber gestanden, wo ich hätte vermuthen müssen, dass die hier erwähnte Fehlerquelle von Einfluss sein würde. Will man jedoch in Zweifelsfällen darauf bedacht sein, Fehlern vorzubeugen, so kann dies dadurch geschehen, dass man zu der abpipettirten Proteïnlösung 1-3 Tropfen 10 procentiger Ammoniumchloridlösung, entsprechend etwa $0,01 \mathrm{~g} \mathrm{\textrm {NH } _ { 4 }} \mathrm{Cl}$, fügt, bevor Quecksilberchlorid zugesetzt wird. In dem oben angeführten Beispiele wird dies darauf hinauslaufen, dass man der Ausfällung von circa $16 \%$ der gesammten Stickstoffmenge vorbeugt, wenn diese als Ammoniaksalze vorhanden sind. Dass hierbei die Protë̈nfällung nicht in besonderem Maasse verändert wird, geht aus den beigefïgten Analysenresultaten hervor. Die Zahlen geben an, wie viele Procente von der gesammten Stickstoffmenge ausgefällt wurden. 
$\$ 54$ Schjerning: Einige kritische Untersuchnngen über die quantitativen

Tabelle III.

\begin{tabular}{|c|c|c|c|c|}
\hline & \multirow{2}{*}{$\begin{array}{l}10 \text { cc Lösung ent- } \\
\text { halten Stickstoff, } \\
\text { entsprechend } \\
\text { Cubikcentimetern } \\
1 / \text { on Normal-Sänre }\end{array}$} & \multicolumn{2}{|c|}{$\begin{array}{c}25 \text { cc Lösung mit } \\
\text { gefällt }\end{array}$} & \multirow{2}{*}{$\begin{array}{l}\text { Zulässiger } \\
\text { Fehler } \\
\text { in } \\
\text { Procenten }\end{array}$} \\
\hline & & $\begin{array}{c}\text { ohne } \\
\mathrm{NH}_{4} \mathrm{CI}\end{array}$ & $\begin{array}{l}\text { nit } 3 \text { Tropfen } \\
\mathrm{NH}_{4} \mathrm{Cl} \\
\text { (10 procentig) }\end{array}$ & \\
\hline Bier . . . . & 2,9 & 19,3 & 17,9 & 1,4 \\
\hline Trubsackwürze . & 6,2 & 19,4 & 18,1 & 0,6 \\
\hline Witte's Pepton... & 9,1 & 25,5 & 25,5 & 0,4 \\
\hline Liebig's Fleischextract & 5,8 & 25,5 & 24,8 & 0,7 \\
\hline Blutserum . . . : . & 6,3 & 81,3 & 81,3 & 0,6 \\
\hline Hefeabsud . . . . . & 3,9 & 42,0 & 40,0 & 1,0 \\
\hline
\end{tabular}

Eine grössere Menge Ammoniumchlorid zuzusetzen, geht nicht an, da die Fällung dann reciprok werden oder sogar gänzlich misslingen würde.

Wie früher gezeigt wurde, sind die Blei- und Quecksilberfällungen identisch; man braucht daher nur eine dieser Fällungen vorzunehmen. Bei der Bleifällung ist es nothwendig, einen zu grossen Ueberschuss des Fällungsmittels zu vermeiden, und, wie ans dem Forhergehenden hervorgeht, muss man bei der Quecksilberfällung auf seiner Hut sein, wenn die vorliegende Proteïnlösung verhältnissmässig grössere Mengen Ammoniumsalze enthält. Jedoch wird es, wie ich glanben möchte, mit Hülfe der hier gegebenen Aufschlüsse und Anweisungen immer möglich sein, mit einem der Fällungsmittel ein zuverlässiges Resultat zu erhalten. In der Regel wird jedoch die Quecksilberfällung der Bleifüllung vorzuziehen sein.

Was die Sättigungsfällung mit $\mathrm{Magnesiumsulfat} \mathrm{anlangt,} \mathrm{so}$ wird aus Tabelle II erhellen, dass nicht wenige stickstoffhaltige organische Stoffe sich aussalzen lassen; so von den von mir gepritiften Hippursäure, Coffeön, ein wenig Theobromin, Chinin, Brucin, Amygdalin and Solanin. Diese. sind sämmtlich als ziemlich schwer lösliche Stoffe anzusehen, von welchen behauptet werden darf, dass sie in der Regel nicht zugegen sein werden, wo es sich um eine vollständige quantitative Bestimmung der verschiedenen Proteïngruppen handelt. Dessenungeachtet muss jedoch bestimmt betont werden, dass man bei der Benutzung dieser Sättigungsfällung sehr behutsam sein muss, wemn zur Untersuchung eine Proteïnlösung vorliegt, welche zugleich einen von diesen und wohl 
mehrere ähnliche Stoffe enthält. In dem Falle ist es nothwendig, sich ausschliesslich an die Fällungen mit Zinnchlorür, Quecksilberchlorid oder Bleiacetat, Ferriacetat und Uranacetat zu halten, wonach es also nur möglich wird, die Mengen von Albumin I, Albumin II + Denuclë̈n, Propepton and Pepton, aber nicht Albumin II und Denuclein einzeln zu bestimmen. Oder man muss versuchen, die Denucleinbestimmung durch Fällung mit Barytwasser, wie dies früher beschrieben wurde ${ }^{1}$ ), vorzunehmen.

Was meine Fällungen angeht, werde ich nur noch die Uranfälluag bei Anwesenheit ron Phosphorsäure erwähnen.

Aus den in Tabelle II verzeichneten Resultaten wird für diese Fällung hervorgehen, dass die gesammte ausgefällte Ammoniakmenge bei mehreren Versuchen betrug diese circa $11 \%$ - durch Eindampfen mit Magnesiamilch quantitativ fortgeschafft wird. Dieses hat sich bei mehreren später angestellten Versuchen bestätigt. Im Uebrigen zeigen die Resultate, dass Uranacetat bei Gegenwart von Phosphorsäure etwas Asparagin, Arginin, bedeutende Mengen Piperazin und eine ganz geringe Menge Brucin ausfällen kann. Bei Anwesenbeit grösserex Mengen Phosphorsäure wird es daher nothwendig sein, bei der Benutzung der directen Uranacetatfällung etwas behutsam zu Werke zu gehen. Eine so grosse Menge Phosphorsäure, wie die hier benutzte, wird jedoch gewiss nur ausnahmsweise vorhanden sein, und in solchen Fällen, wo es sich zugleich darum handelt, eine quantitative Bestimmung der gesammten Proteïnmenge zu erhalten, kann ein völlig zuverlässiges Resultat auf einem kleinen Umwege erreicht werden, indem zuerst eine Fällang bei $50^{\circ} \mathrm{C}$. mit einer nicht ganz ausreichenden Menge von Bleizetat vorgenommen wird, wodurch vatürlich die Phosphorsäure, zu:ammen mit einigen Proteïnen, fast vollständig ausgefällt wird. Das Filtrat von der Bleifällung wird dann auf die gewöhnliche Weise mit Jranacetat gefällt. Die gesammte Stickstoffmenge in den ausgevaschenen Blei- und Uranfällungen ist dann gleich der gesammten 'roteïnstickstoffmenge. Nachfolgende Tabelle wird die Richtigkeit dieses rerfahrens zeigen. Die Zahlen geben wie gewöhnlich an, wie viele rocente der gesammten Stickstoffmenge ausgefällt wurden.

1) Diese Zeitschrift 33, 271; 34, 137-139. 
556 Schjerning: Einige kritische Untersuchungen über die quantitativen

Tabelle IV.

\begin{tabular}{|c|c|c|c|c|}
\hline$\because=-=$ & $\left\{\begin{array}{l}10 \text { cc Lösung ent- } \\
\text { balten Stickstofi, } \\
\text { entsprechend } \\
\text { Cubikcentimetern } \\
1 / 10 \text { Normal-Säure }\end{array}\right.$ & $\begin{array}{l}25 c c \text { Lösung } \\
\text { gefällt mit } \\
\text { Uran auf } \\
\text { gewöhn- } \\
\text { liche Weise }\end{array}$ & $\begin{array}{c}25 c c \text { Losung } \\
+0.04 \mathrm{~g} \mathrm{P}_{205} \\
\text { getailt mit } 3 c c \\
\text { 10procent ger } \\
\text { Bleiacetat- } \\
\text { lösnng. Das } \\
\text { Filtrat gefallit } \\
\text { mit Uran. }\end{array}$ & $\begin{array}{l}\text { Zulässiger } \\
\text { Eehler } \\
\quad \text { in } \\
\text { Procenten }\end{array}$ \\
\hline Witte's Pepton & 9,1 & 51,4 & $51,01)$ & 0,4 \\
\hline Liebig's Fleischextract & 5,8 & 38,6 & 38,6 & 0,7 \\
\hline Hefeabsud . . . . & 3,9 & 60,5 & 59,5 & 1,0 \\
\hline Trubsackwürze . . & 6,2 & 34,2 & 32,9 & 0,6 \\
\hline
\end{tabular}

Die hier erwähnte Bleifällung unter Kochen vorzunehmen, ist nicht räthlich, da man dabei leicht die Peptone und etwa auch die Propeptone einer theilweisen Spaltung aussetzt. Aus dem nämlichen Grunde möchte ich nicht vorschlagen, die Uranfällung in dem. Filtrate von der normalen Bleiacetatfällung vorzunehmen, was allerdings weitaus am einfachsten wäre. Wie aus dem ersten Versuche - Witte's Pepton -, wo die Bleifällung sich nicht auf einem Filter sammeln lässt, erhellt, wird es kaum nothwendig sein, die Bleifällung für sich zu sammeln, sondern man kann unmittelbar, nachdern man Bleiacetat zugesetzt hat, auf die gewöhnliche Weise direct mit Uranacetat fällen. Dieses Verfahren habe ich jedoch nicht näher geprüft, da die Mühe, welche damit verbunden ist, den Bleiniederschlag für sich zu sammeln, mit Wasser auszuwaschen und danach im Filtrate mit Uranacetat zu fällen, so gering ist, dass man in den seltenen Fällen, wo mit verhältnissmässig phosphorsäurereichen Proteinlösungen gearbeitet wird, kaum Anstand zu nehmen braucht, sich ihr zu unterziehen.

Was das Verhalten der übrigen Proteïnfüllungsmittel gegenüber nicht-proteïnartigen organischen Stickstoffverbindungen anlangt, erwähne ich zuerst:

Die Fällung mit Bromwasser in schwach salzsaurer Flüssigkeit.

1) Die Bleifällung liess sich nicht abfiltriren, weshalb die Uranfällun६ gleich nach Zugabe der Bleiacetatlösung vorgenommen wurde. Das Ergebnis zeigt, dass die Phosphorsäure trotzdem unwirksam gemacht ist (vergleich Tabelle V). 
Aus der Tabelle II geht hervor, dass diese Fällung ungefähr meiner Sättigungsfällung mit Magnesiumsulfat in saurer Flüssigkeit gleichkommt, indem Brom fast ganz dieselben Stoffe fällt wie Magnesiumsulfat. Kommt nun noch hinzu, dass die Bromfüllung sich sehr oft schwer auf einem Filter sammeln lässt, - die Niederschläge sind oft so fein vertheilt, dass sie leicht durch die Poren des Filters gehen, selbst wenn ein wenig Magnesiumsulfat zugesetzt wird -, so zeigen also schon diese Versuche, dass man bei Benutzung dieser Fällung auf keine besonders grosse Genauigkeit rechnen darf. Wie Brom sich gegenüber den 'wirklichen Proteïnstoffen verhält, wird später erwähnt werden.

Gerbsäure als Proteïnfällungsmittel zu benutzen, wenn es sich darum handelt, nicht zugleich nicht-proteïnartige stickstoffhaltige Stoffe ausgefällt zu bekommen, scheint nach Tabelle II gerechtfertigt, indem bloss Neurin damit eine wirkliche Fällung gibt. Andererseits wird es doch wohl keinem $Z$ weifel unterliegen, dass auch Coffeïn, Chinin, Brucin and Solanin durch die Gerbsäure ausgefällt werden, wenn diese Stoffe zusammen mit Proteïustoffen vorkommen, indem dann die mit den genannten Stoffen gebildeten, fein vertheilten Ausscheidungen mit niedergerissen und zusammen mit der Proteïnausscheidung auf dem Filter gesammelt werden.

In seinem Verhalten gegenüber nicht-proteïnartigen Stoffen gleicht Stutzer's Reagens sehr dem Ferriacetat.

Wie die Tabelle II zeigt, ist die Kupferfällung jedoch viel weniger zuverlässig als meine Ferriacetatfällung. Wenn die Fällung auf die früher erwähnte Weise ausgeführt wird, zeigt das Reagens jedoch nur sehr geringe Neigung, die am häufigsten vorkommenden Aminsäuren auszufällen, wogegen recht bedeutende Mengen Guanin, Alloxan, Harnsäure nebst ein wenig Arginin, ganz wenig Allantoin, Kreatin und Chinin ausgefällt werden. Will man dagegen bei gewöhnlicher Temperatur die Fällung vornehmen, oder sie über einen längeren Zeitraum ausdehnen, so zeigt Tabelle I, dass man bei der Kupferfäilung nicht so günstige Resultate erwarten darf wie die in Tabelle II verzeichneten.

Die Anwendbarbeit der Phosphorwolframsäure als Proteïnfällungsmittel scheint schon in der vorliegenden Litteratur recht zweifel- 
558 Schjerning: Einige kritische Untersuchungen über die quantitativen

haft. ${ }^{1}$ ) Nichtsdestoweniger wird sie neuerdings von Mallet ${ }^{2}$ ) als quantitatives Fällungsmittel für Proteïnstoffe verwendet. Eine Sonderstellung nimmt Laszczynski in seiner oben citirten Abhandlung ein, indem er darüber im Klaren ist, dass dieses Reagens auch andere organische stickstofthaltige Stoffe ausfällt als die Proteïne, während er doch, bei seinen Untersuchungen über Peptase, trotzdem eben dasselbe Reagens gerade als Proteïnfällungsmittel benutzt.

Aus den Versuchen, welche ich über Phosphorwolframsäure angestellt habe - Tabelle II -, geht hervor, dass dieses Reagens nicht als quantitatives Fällungsmittel für Proteïnstoffe - wenn zugleich andere Stickstoffverbindungen vorhanden sind - benutzt werden soll, da gleichzeitig eine sehr bedeutende Anzahl nicht-proteïnartiger organischer Stickstoffverbindungen ausgefällt werden, und zwar zumeist in recht erheblichen Nengen. Selbst wenn diese Fällung unter Kochen vorgenommen wird, fält sie doch für die nicht-proteïnartigen Stoffe bei weitem nicht zufriedenstellend aus, und, wie ich später zeigen werde, wird in Folge dessen die Proteïnfällung selbst theilweise verfehlt.

\section{II.}

Soll indessen eine kritische Beurtheilung der verschiedenen Proteïnfällungsmittel vorgenommen werden, so ist es nothwendig, eine ähnliche Reihe von Versuchen mit verschiedenen Proteïnlösungen anzustellen, wie sie im Vorhergehenden mit nicht-proteïnartigen Stoffen ausgeführt wurde.

Ausser den früher benutzten und beschriebenen Fallungen wurden bei dieser Reihe auch mit der von Laszczynskiª ${ }^{3}$ angegebenen Methode Versuche gemacht. Diese besteht ganz einfach darin, dass die Proteïnlösung 1 Stunde lang bei $1 \frac{1}{2}$ Atmosphären Druck erhitzt wird, wonach das gebildete Coagulum sogleich auf einem Filter gesammelt und 2-3 mal mit warmem Wasser ausgewaschen wird. Die Resultate dieser Versuche sind in der nachstehenden Tabelle $\mathrm{V}$ angegeben. Die Zahlen bezeichnen wie gewöhnlich Procente der gesammten Stickstoffmenge.

1) E. Bosshard, diese Zeitschrift 22, 329; Hedin, Zeitschr. f. physiol. Chemie 21, 160.

2) The Analyst 1898, S. 328 .

3) Zeitschrift für das gesammte Brauwesen 22, 124 und 141. 
Fällungsverhältnisse verschiedener Proteünfällungsmittel.

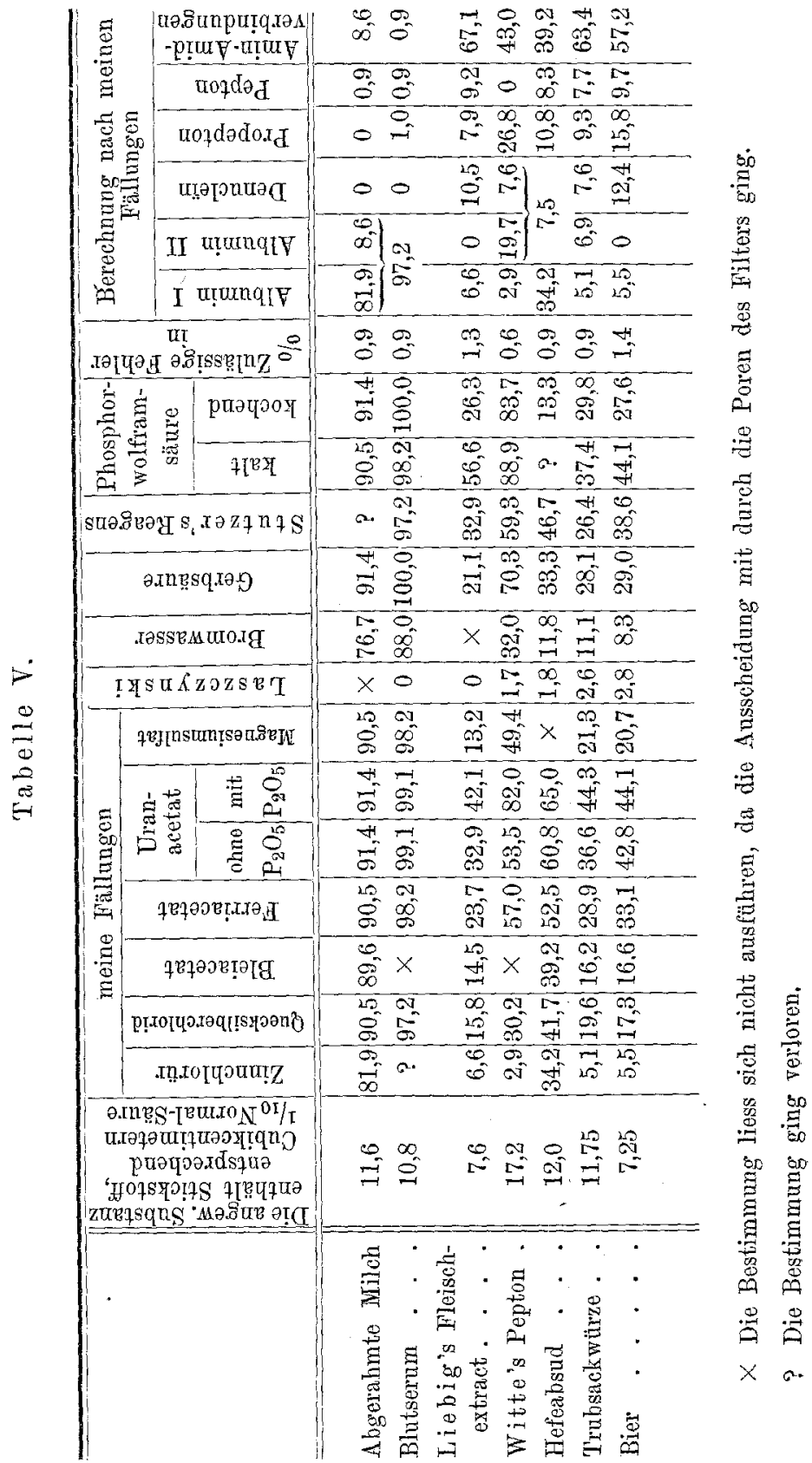


560 Schjerning: Einige kritische Untersuchungen über die quantitativen

Um aus diesen Versuchen die Resultate abzuleiten, wird es nicht nothwendig sein auf meine Fällungen näher einzugehen, da ähnliche Versuche mit eben denselben Proteïnlösungen früher ausgeführt und besprochen wurden - siehe meine diesbezüglichen Publicationen. Der Schluss der Tabelle $V$ (die letzten 6 Colonnen) zeigt die Mengen der verschiedenen Proteïngruppen, nach meiner Methode berechnet — aus den Fällungen mit Zinnchlorür, Quecksilberchlorid, Ferriacetat, Uranacetat ohne Phosphorsäure und Magnesiumsulfat. Es sei nur noch bemerkt, dass die Fällung mit Uranacetat nach Zusatz von Phosphorsäure zur Evidenz zeigt, dass die Gegenwart grösserer Mengen Phosphorsäure in den meisten Fällen, wo die Proteïnlösung ausser wirklichen Protë̈nen zugleich merkbare Mengen nicht - proteïnartiger organischer Stickstoffverbindungen enthält, bewirkt, dass ausser den wirklichen Proteïnen auch noch nicht unerhebliche Mengen von den nicht-proteïnartigen Stoffen ausgefällt werden. Dass dieser Fehler jedoch im gegebenen Falle vermieden werden kann, und auf welche Weise, ist in Tabelle IV gezeigt.

Von den übrigen Fällungen kommen wir zuerst zu der Laszczynski'schen. Von vorn herein liess es sich allerdings denken, dass eine so starke Temperatureinwirkung - 1 Stunde langes Erbitzen bei $1 \frac{1}{2}$ Atmosphären - eine Spaltung der verschiedenen Proteïnmolecüle zur Folge haben müsste; persönlich war ich.zu der Annahme geneigt, dass man auf diese Weise das Auscoaguliren aller wirklichen Albumine erzielen würde. Die in Tabelle $V$ angegebenen Resultate zeigen jedoch, dass dem keineswegs so ist. Schon die mit Milch und Blutserum erhaltenen Resultate zeigen deutlich genug, dass dieser Methode keine allgemeine Gültigkeit zukommt, und betrachten wir die übrigen Versuchsobjecte etwas näher, so stellt sich herans, dass nur äusserst geringe Mengen oder gar keine Albumine auscoaguliren, während doch thatsächlich grössere oder geringere Mengen wirklicher Albumine vorhanden sein müssen

Ein näheres Eingehen auf die Sache bestätigte die Richtigkeit de] Annahme, dass in Folge dieser ziemlich starken Wärmeeinwirkung ein tiefer gehende Spaltung der Albuminmolecüle stattfinden würde, indem wie Tabelle VI zeigt, eine Quecksilberfällnng ausgeführt in dem Filtrat von Laszczynski's Fällung stets ein niedrigeres Resultat gab, al nach der Natur der Sache zu erwarten war. 
Tabelle VI.

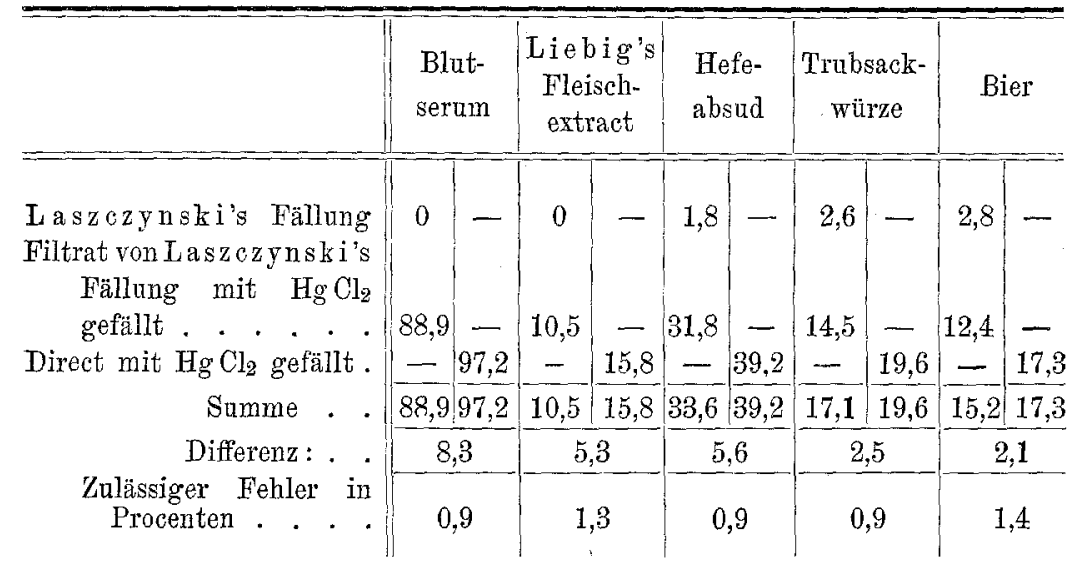

Nach diesen Ergebnissen zu urtheilen, ist es das Eiweissmolecül selbst, das eine Veränderung - etwa eine Peptonisirung - erleidet. Bei den drei Versuchen mit Fleischextract, Trubsackwürze und Bier, bei welchen nur eine verhältnissmässig geringe Menge wirklicher Albumine vorhanden ist, ist es bemerkenswerth, dass die oben angegebene Differenz, zu Laszczynski's Fällung addirt, ungefähr dieselbe Zahl gibt wie meine Fällung mit Zinnchlorür (siehe Tabelle V), was darauf hindeutet, dass auf Albumin I eine besonders starke Einwirkung ausgeübt wird.

Noch eine andere Betrachtung möge bei dieser Fällung ihren Platz finden, nämlich die, dass es für das quantitative Resultat unmöglich gleichgültig sein kann, ob die vorliegende Proteïnlösung neutral, sauer oder alkalisch reagirt, oder ob sie grössere oder geringere Mengen verschiedener mineralischer Stoffe enthält. Wie ältere Versuche und Erfahrungen zeigen ${ }^{1}$ ), sind diese Factoren von sehr wesentlicher Bedeutung, wenn es sich um das Auscoaguliren von Eiweissstoffen handelt. Auf Grund des hier Gesagten kann der Laszczynski'schen Methode keine allgemeine analytische Bedeutung beigelegt werden.

Nicht besser ist es um die Bromfällung bestellt. Selbst wenn ein wenig Magnesiumsulfat zugesetzt und der sich bildende Niederschlag mit verdünntem Bromwasser ausgewaschen wird, genügt diese

1) E. Varenne, Berichte der deutschen ehem. Gesellschaft 19, Ref. 356, nach Bull. soc. chim. 45, 427; E. Harnack, Berichte der deutschen chem. Gesellschaft 22, 3046.

Fresenius, Zeitschrift f. analyt. Chemie. Xxxrx. Jahrgang. 9. Heft. 
Fällung oft nicht einmal der bescheidenen Forderung ein klares Filtrat zu geben. Betrachtet man ferner die Milch und Blutserum betreffenden Resultate, von welchen beiden Proteïnlösungen wir mit. Sicherheit wissen, dass sie beinahe die ganze Stickstoffmenge als Proteïne enthalten, so karin es keinem Zweifel unterliegen, dass die Bromfällung sich bei der quantitativen Trennung von Proteïnstoffen (Albuminen, Albumosen, Peptonen) von anderen stickstoffhaltigen, organischen Stoffen - Nicht-Proteïnen - gar nicht anwenden lässt, wie das von Allen und Searle behauptet wurde.

Wie bekanut bilden Chlor, Brom und Jod durchgängig schwer lösliche Verbindungen mit den Albuminen und möglicherweise auch mit anderen Proteïnstoffen; etwas ganz Aehnliches ist aber auch mit nichtproteinnartigen Stickstoffverbindungen (Chinin) der Fall. ${ }^{1}$ ) Von diesen Thatsachen zur Anwendung der Bromfällung bei der quantitativen Proteïnanalyse scheint — jedenfalls für die von Allen und Searle gegebene Form - nach meinen Versuchszahlen ein weiter Sprung zu sein.

In Beziehung auf die Gerbsäurefällung gelange ich der Hauptsache nach zu demselben Resultat wie Sebelien. ${ }^{2}$ ) Albumosen (Propeptone) und wirkliche Peptone werden entweder gar nicht oder jedenfalls nur theilweise gefällt, und wenn hierzu noch kommt, dass wenigstens einige nicht-proteïnartige, organische Stickstoffverbindungen auch von Gerbsäure ausgefállt werden können, scheint diesem Fällungsmittel in der gewöhnlichen quantitativen Proteïnanalyse keine sehr grosse Bedeutung zuzukommen. Nur wirklichen Albuminen gegentiber ist die Gerbsäurefällung absolut quantitativ; sobald aber andere Proteïngruppen vorhanden sind, wird die Fällung zweifelhaft.

Stutzer's Kupferfällung. Im Laufe der Jahre hat diese Fällung eine weite und wohlberechtigte Verbreitung und Anwendung gewonnen, und wenige Fällangsmittel sind wie dieses nach und nach gegenüber so vielen verschiedenartigen Stoffen geprüft worden. Die natürliche Folge hiervon ist die gewesen, dass von verschiedenen Seiten auf die die Methode begleitenden Fehlerquellen aufmerksam gemacht wurde. Diese gipfeln in zwei Punkten, nämlich dass das Reagens Amin-Amidverbindungen - als schwerlösliche Kupfersalze -- ausfällen

1) Chri istensen, D. kgl. danske Vidensk. Selsk: 6 Riekke IX, 5, pag. 253.

2) Diese Zeitschrift 28, 383. 
kann, und dass es die wirklichen Peptone nicht mit quantitativer Genauigkeit fällt.

Wenn berucksichtigt wird, wie schwer löslich die Krpfersalze mehrerer Aminsäuren sind, liegt es nahe, vor der Metbode zu warnen, jedenfalls wenn es sich darum handelt, die Proteïnstoffe in einer Lösung, welche zugleich beträchtliche Mengen Aminsäuren enthält, zu bestimmen. Meine Versuche - Tabelle II - führen mich indess zu dem gleichen Resultat wie das von E. Schulze erlangte, ${ }^{1}$ ) dass diese Fehlerquelle in der Regel eine ganz untergeordnete Rolle spielen wird, wenn die Fällung nur in der möglichst kürzesten Zeit, bei passender Temperatur and in einem reichlichen Volumen Flüssigkeit vorgenommen wird. Werden diese Regeln überschritten, so nimmt nach der Natur der Sache auch die quantitative Zuverlässigkeit der Fällung ab.

Nein, der besondere Haupteinwand gegen die Stutzer'sche Fällung ist der, dass sie nicht mit quantitativer Sicherheit die wirklichen Peptone ansfällt, welche doch, wenn es sich um die gesammte Proteïnmenge handelt, eine recht wesentliche Rolle als stickstoffhaltige Nahrungsstoffe spielen. Eine Betrachtung der Tabelle $V$ zeigt, dass die Kupferfällung an Quantität zumeist der Ferriacetatfällung sehr nahe, aber nur ausnahmsweise der Uranfällung gleich kommt, jedenfalls immer danm, wenn die betreffende Proteinlösung wirkliche Peptone enthält. Da es nun aus Tabelle II recht deutlich hervorgeht, dass weder Ferriacetat noch Uranacetat unter normalen Verhältnissen sehr geneigt sind, Amin-Amidverbindungen auszufällen, und da das Verhältniss zwischen den Ferriacetatund Uranfällungen früher erläutert wurde, muss auch der Schluss berechtigt sein, dass Stutzer's Reagens wirkliche Peptone nicht oder doch nur theilweise ausfällt.

Um dieses noch klarer zu beweisen, bemerke ich, dass in Bezug auf das Verhältniss der Kupferfällung zu meinen Fällangen nur zwei Möglichkeiten denkbar sind - vorausgesetzt, dass die Fâllung überhaupt Punkt II Seite 545 erfüllt, nämlich:

I. dass sie entweder Albumine sammt Denucleïnen und Propeptonen enthält, also mit meiner Ferriacetatfällung vollkommen identisch sein muss,

1) Landw. Versuchs-St. 26, 213; 27, 449; 33, 124. 
564 Schjerning: Einige kritische Tntersuchungen über die quantitativen

II. oder dass sie Albumine sammt Propeptonen und wirklichen Peptonen enthält, mit anderen Worten den Fällungen Magnesiasulfat + (Uranacetat $\div$ Ferriacetat), nach meiner Arbeitsweise, entsprechen muss.

Die Resultate einer Reihe von Versuchen mit Malz, Würze, Bier und Liebig's Fleischextract, welche nicht hier einzeln mitgetheiit werden sollen, sondern bloss unten als Durchschnittszahlen sämmtlicher Bestimmungen ausgedrückt sind, zeigen, wie die früheren Versuche, dass der ersten Möglichkeit die grössere Wabrscheinlichkeit zukommt.

Albumin + Denucleïn + Propepton 29,8\% des gesammten Stickstoffs, Albumin + Propepton + Pepton

Stutzer's Fällung

Zulässiger Fehler

$$
\begin{aligned}
& 28,9 \% \ll \quad \ll \\
& 31,3 \% \quad \ll \quad \ll \quad \text { « } \\
& 0,9 \% \quad \ll \quad \ll
\end{aligned}
$$

Volle Gewissheit für die Richtigkeit erlangt man jedoch erst durch nähere Betrachtung des in Tabelle $V$ angeführten Versuches mit Witte's Pepton - eine Proteïnlösung, welche gar keine wirklichen Peptone enthält - indem sich hieraus berechnen lassen:

Albumin + Denucleïn + Propepton 57,0\% des gesammten Stickstoffs, Albumin + Propepton + Pepton Stutzer's Fällung $49,4 \%<$
$59,3 \%<$

Auf Grund dieses Versuches wird die Behauptung, dass Stutzer's Reagens die wirklichen-Peptone nicht fälle, erst völlig berechtigt, ein Resultat, zu welchem schon früher $\mathrm{H}$. Weisk $\mathrm{e}^{1}$ ) und A. Stutzer ${ }^{2}$ ) gelangten.

Wo es sich daher darum handelt, die Menge der verschiedenen Proteïngruppen quantitativ zu bestimmen, wird Stutzer's Fällung nicht genügen, wohl aber, wo es sich lediglich um Bestimmung der gesammten Proteïnmenge in einem Stoffe, welcher keine wirklichen Peptone und nicht verhältnissmässig grosse Mengen Amin-Amidverbindungen enthält, handelt.

Was die Phosphorwolframsäure als Proteïnfällungsmittel anbelangt, so muss ich bei meinem früheren Ausspruche, dass die Phosphorwolframsätre gleich wie das Uranacetat alle Proteïnstoffe fälle ${ }^{3}$ ), stehen bleiben.

1) Landw. Versuchs-Stationen 33, 147.

2) Diese Zeitschrift 31, 505 .

3) Diese Zeitschrift $230,295$. 
In sehr vielen Fällen kann indess die Phosphorwolframsäurefällung gar nicht benutzt werden, da auch zahlreiche nicht-proteïnartige, stickstoffhaltige Stoffe gleichzeitig in grösserer oder geringerer Menge ausgefällt werden. Diesen Fehler durch Vornahme der Fällung in kochender Flüssigkeit zu vermeiden, scheint nur theilweise möglich (siehe Tabelle II), und wirft man einen Blick auf Tabelle $V$, so wird sich herausstellen, dass man hierbei zu einem in mehreren Fällen recht erheblichen neuen Fehler Anlass gibt. Es scheint sich nämlich so zu verhalten, dass die Phosphorwolframsäure, wenn die Fällung in kochender Fltussigkeit vorgenommen wird, nicht oder doch nur theilweise wirkliche Peptone - und möglicherweise Albumosen - ausfällt, sei es nun dass dieses Verhalten von einer tiefer gehenden Spaltung der betreffenden Molecutle oder von einer grösseren Löslichkeit der Phosphorwolframsäureverbindungen herrührt!

Aus meinen Versuchen mit Milch und Blutserum - Proteinlösungen, welche fast die ganze Stickstoffmenge in Form wirklicher Albumine enthalten - geht hervor, dass -- in vollkommener Uebereinstimmung mit dem oben Gesagten - sowohl die kalte als die kochende Fällung ganz identisch mit der normalen Uranfällnng siñd. Die Versuche mit den übrigen Proteïnlösungen - welche nur eine grössere oder geringere Menge des gesammten Stickstoffgehaltes in Form von Albuminen, aber dagegen recht bedeutende Mengen als Albumosen, wirkliche Peptone und Amin-Amidverbindungen enthalten - zeigen dagegen durchgehends, dass die kalte Phosphorwolframsäurefällung grösser (für Witte's Pepton und Fleischextract) oder eben so gross (für Würze und Bier) als die normale Uranfällung ist, während die kochende Fällung stets ein niedrigeres Resultat gibt als die kalte. Auch diese Ergebnisse stimmen mit dem oben Angeführten vollkommen überein.

Wenn also die kalte Phosphorwolframsäurefällung in Trubsackwürze und Bier dasselbe Resultat gibt wie die normale Uranfällung, so muss dies davon herrühren, dass diese beiden Proteünlösungen nicht oder doch nur in sehr geringer Menge nicht-proteinnartige, stickstoffhaltige, mit Phosphorwolframsäure fällbare Stoffe enthalten. Das Gegentheil muss mit Witte's Pepton, Liebig's Fleischextract und wahrscheinlich vielen anderen Stoffen der Fall sein.

Davon, die kochende Phosphorwolframsänrefällung zu benutzen, ist entschieden abzurathen, und wenn man die Fällung bei gewöhnlicher Temperatur vornimmt, muss man sich erinnern, dass zahlreiche nicht- 
proteïnartige Stickstofiverbindungen gleichzeitig ganz oder theilweise ausgefällt werden können. Aus dem letzterwähnten Grunde eignet sich die Phosphorwolframsäure nicht als quantitatives Proteïnfällungsmittel, wogegen sie in der präparativen Chemie vorzügliche Dienste leisten kann.

Bezüglich der Proteïnfällungen überhaupt will ich nur noch bemerken, dass, wenn der zur Untersuchung vorliegende Stoff verhältnissmässig grosse Mengen mineralischer Salze leichter Metalle enthält, es nicht möglich sein wird, eine quantitative Bestimmung der verschiedenen Proteïngruppen mit einiger Sicherheit vorzunehmen, weil in diesem Falle die sich bildenden Proter̈n-Metallverbindungen sich reciprok mit den Salzen der leichten Metalle umsetzen und leicht lösliche ProteïnVerbindungen der leichten Metalle bilden. Nur die Sättigungsfällung (mit $\mathrm{MgSO}_{4}$ ), die Fällung mit Zinnchlorür und wahrscheinlich die Fällungen mit Stutzer's Reagens und der Gerbsäure bilden eine Ausnahme hiervon.

Kopenhagen, Juli 1900 .

\section{Mittheilungen aus dem chemischen Laboratorium Fresenius zu Wiesbaden}

Bemerkungen zu dem Abegg-Herz'schen systematischen Gang zur Erkennung und Trennung der Säuren.

Von

\section{W. Fresenius.}

In ihrem $\gg$ chemischen Praktikum ${ }^{1}$ ) sowie in der Zeitschrift für anorganische Chemie ${ }^{2}$ ) haben R. Abegg und W. Herz eine Tabelle zur Erkennung und Trennung der häufigsten Anionen mitgetheilt, welche sie an letzterer Stelle mit der Bemerkung veröffentlichen, dass es bis-

1) Chemisches Praktikum, experimentelle Einführung in präparative und analytische Arbeiten auf physikalisch-chemischer Grundlage ron Dr. R. A bega und Dr. W. Herz, Göttingen, Vandenhoeck und Ruprecht 1900.

2) Zeitschrift $f$. anorganische Chemie, 23, 236. 